\title{
Flawless Detection of Herbal Plant Leaf by Machine Learning Classifier Through Two Stage Authentication Procedure
}

\author{
J. Samuel Manoharan, \\ Professor, \\ Department of Electronics \& Communication Engineering, \\ Sir Isaac Newton College of Engineering and Technology, \\ Nagapattinam, India. \\ Email id: drjsm1530@ieee.org
}

\begin{abstract}
Herbal plants are crucial to human existence for medical reasons, and they can also provide free oxygen to the environment. Many herbal plants are rich in therapeutic goods and also it includes the active elements that will benefit future generations. Many valuable plant species are being extinguished and destroyed as a result of factors such as global warming, population growth, occupational secrecy, a lack of government support for research, and a lack of knowledge about therapeutic plants. Due to the lag of dimensional factors such as length and width, many existing algorithms fail to recognize herbal leaf in all seasons with the maximum accuracy. Henceforth, the proposed algorithm focuses on the incomplete problems in the datasets in order to improve the detection rate for herbal leaf identification. The inclusions of dimension factors in the datasets are performing good results in the image segmentation process. The obtained result has been validated with a machine learning classifier when combined with ex-or gate operation is called deep knowledge-based identification. This two-stage authentication (TSA) procedure is improving the recognition rate required for the detection of herbal leaf. This fusion of image segmentation with machine learning is providing good robustness for the proposed architecture. Besides, intelligent selection of image segmentation techniques to segment the leaf from the image is improving the detection accuracy. This procedure is addressing and answering the drawbacks associated with the detection of the herbal leaf by using many Machine Learning (ML) approaches. Also, it improves the rate of detection and minimizes
\end{abstract}


Journal of Artificial Intelligence and Capsule Networks (2021)

Vol.03/ No.02

Pages: 125-139

http://irojournals.com/aicn/

DOI: https://doi.org/10.36548/jaicn.2021.2.005

the classification error. From the results, it is evident that the proposed method has obtained better accuracy and other performance measures.

Keywords: Herbal Plant Identification, Edge Detection, Machine Learning

\section{INTRODUCTION}

Herbs have been exploited by indigenous people as traditional remedies since prehistoric times. Plants are extremely important in the world for a variety of reasons. Practitioners generally identify herbs based on their age of sensory experience [1]. Recent developments in analytical technologies have significantly contributed to the herbal data recognition. This facilitates many people, in particular those without herbal recognition experience. In addition to the time-consuming procedures, laboratory-based testing requires competence in sample treatment and data interpretation [2].

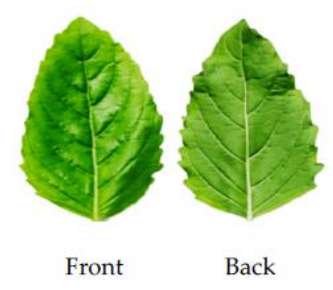

Figure 1 Front and back of Tulsi leaf in the dataset

The ability to identify the leaves of herbal plants in a simple and reliable manner is critical right now. Computing through probability and statistical analysis is most likely a strong technique for accurately classifying plants. To quickly identify herbal leaf items using a nondestructive manner, the method of identification of herbal leaf options is recommended. This method is particularly useful for individuals, who cannot afford to use expensive analytical instruments [3]. Generally, all we know the various herbal leaves namely as follows;

1. Tulsi

2. Omavalli

3. Neem 
Journal of Artificial Intelligence and Capsule Networks (2021)

Vol.03/ No.02

Pages: 125-139

http://irojournals.com/aicn/

DOI: https://doi.org/10.36548/jaicn.2021.2.005

4. Vana Thulasi

5. Thudhuvalai

6. Lime

In some cases, the color and shape characteristics of the herbal plants have been used to obtain the characteristic vectors, where the classification has been performed. Different classification algorithms are utilized for the classification of plants based on the functional vectors. Some recent research papers discuss about the Support Vector Machine (SVM) algorithm for implementing a highly accurate herbal plant identification method [4]. Recently, many algorithms are discussed and that the distribution of the Gauzian leaf features will provide higher efficient classificatory.

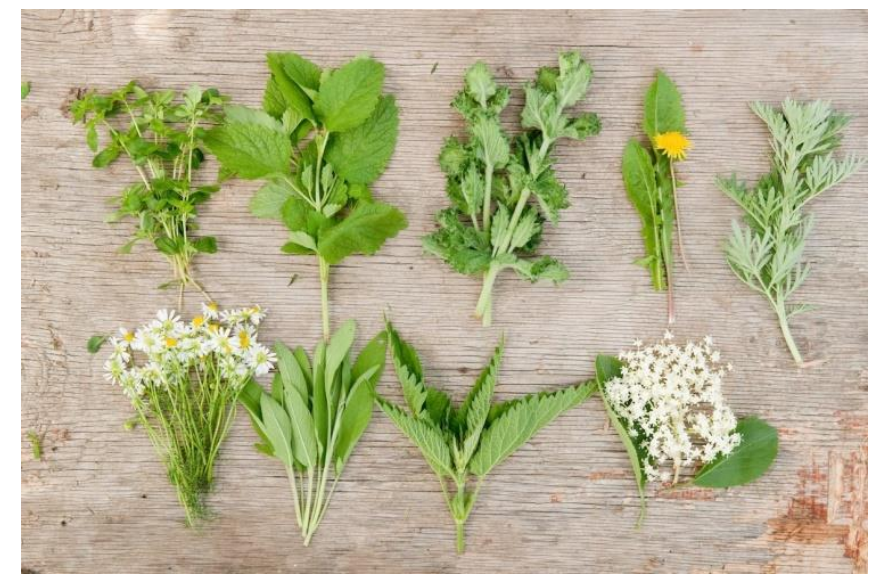

Figure 2 Sample images of herbal plants

Also, the research papers will further investigate the research methods for attaining better classification by implementing different algorithms, such as probabilistic statistical neural network, supporting vector machine, main component analysis, and texture base analysis used to detect a plant. Many authors only consider the leaves of plants for plant identification because the leaves are two-dimensional in nature and are always available $[5,6]$. 


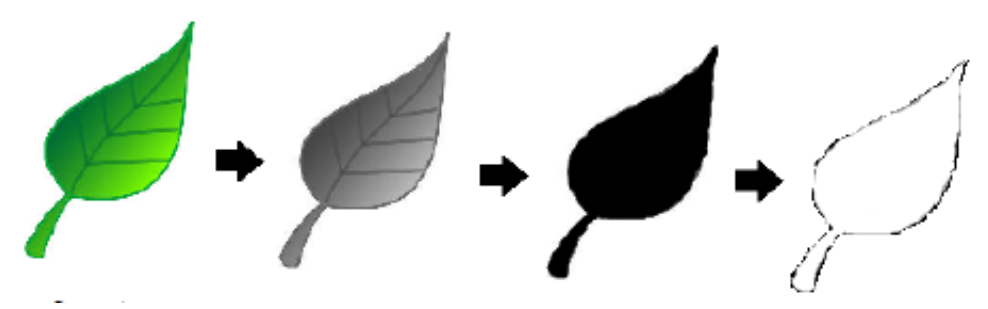

Figure 3 Herbal Plant Leaf Pre-Processing Stage

The image pre-processing stage includes grey scale conversion with binary transformation. Finally, edge detection will be performed using a variety of algorithms, as illustrated in Figure 3. However, because flowers and fruit/sowing are 3D in nature and only available during particular seasons, only less research study is available for identifying the medicinal plants. This paper will briefly examine the identification and classification of medicinal plants using various technologies in the pre-processing, feature extraction, and classification phases $[7,8]$.

\section{ORGANIZATION OF THE RESEARCH}

The workflow of the research article is well structured as follows: Section 3 gives recent research on herbal leaf identification. The proposed identification work will be discussed in Section 4. Section 5 showed and discussed the results obtained from the exam. Section 6 deals with the conclusion and future task.

\section{PRELIMINARIES}

Surya. C et al identified the 20 Ayurvedic frontal and rear leaves of 40 different species. The Weka tool is used to identify medicinal plants with algorithms for machine education. Leaves are extracted from the collection of colors and binary images, color, and texture features. Supporting classifiers of the Vector Machines (SVM) and the MLP classifiers are used to identify blades based on the many characteristics of Geometric distances, centroid radii, color traits, texture traits, HU invariant moments, and Zernike moments. They achieved better results through Vector Machine Support (SVM) [9, 10]. 
Journal of Artificial Intelligence and Capsule Networks (2021)

Vol.03/ No.02

Pages: 125-139

http://irojournals.com/aicn/

DOI: https://doi.org/10.36548/jaicn.2021.2.005

Adams Begue et al. recommended a way to automatically identify medicinal plants using machine learning techniques using leaf characteristics. For classification purposes, five different classifiers are used. Of these, the better accuracy is achieved from the random forest classifications machine learning approach which is compared with neighbors nearest to $\mathrm{k}$, naive bays, SVM, and neural networks $[11,12]$.

Zhou et al discuss the formal features and textures of the blades that are considered to identify and classify the medicinal plants within the Traditional Chinese Medicine (TCM) system $[13,14]$. Riddhi $\mathrm{H}$ et al talk over about extracted image features that are fed into the classification of the Support Vector Machine (SVM). Gray-level Co-occurrence Matrices (GLCM) and Color moment extract the texture and color of flowers [15]. Features extracted are fed into the classification systems of the Neural Network. GLCM and Color moment are individually accurate at low. The hybrid precision combination is higher than other existing methods [16].

Marco Seeland et al focus on the local features from the flowers and it is classifying the flora based on the color and form characteristics, the authors considered three different datasets in the name with oxford flower. For fusion, pooling, extraction, and detection of flowers, different methods are employed. They concluded that Jena Flower 30 dataset is the highest rating accuracy of 94 percent compared to two other data sets [17].

Pradeep Kumar et al developed an automatic system for the identification of fruit using edge and color-based segmentation methods. $\mathrm{K}$ means the clusters and detection of the canny edge methods are performing in the fragmentation of the image. The algorithm based on color outperforms and produces 85 percent of the algorithm based on edges [18].

Venkataraman D et al reviewed classification methods to identify medicinal plants, including probabilistic neurological network, vector support machine, and principal component analysis, which has been carried out by authors in the paper. The form, color, and vein properties of the neam leaves and the Aspect Ratio, the Centroid, the area, the perimeter, and the roundness characteristic of the leaf were calculated in their proposed work [19]. 
Journal of Artificial Intelligence and Capsule Networks (2021)

Vol.03/ No.02

Pages: 125-139

http://irojournals.com/aicn/

DOI: https://doi.org/10.36548/jaicn.2021.2.005

Ruaa Adeeb et al compare various machine learning algorithms for fruit identification that the fruit form, color, and texture skins to develop a system for fruit recognition with higher accuracy. Three different classes, the $\mathrm{K}$ - Nearest neighbor (k-NN), binary classification tree, and support vector machine, have been trained for these extracted features (SVM). SVM classification's performed best results with 100 percent accuracy [20].

Sana $\mathrm{O}$ et al developed an android application for the mobile identification of herbal plants through texture extraction techniques. This system identifies the medicinal plants based on a confirmed herbal plants picture. Co-accident matrices are used for texture extraction and image processing techniques of the classification of plants. The device is free, saves time and costs, and does not need expert assistance. It is maintained the identification process and query of plant species information in the botanical gardening, medical and cosmetic industries [21].

Many research papers are focused on classification methods to detect herbal plant leaves without any classification error. But many algorithms are failed to attain those compromises due to incomplete dataset, choice of inappropriate image segmentation. Because the herbal plant leaf sizes are variable and the same segmentation algorithm is not suitable for the highest prediction. But our algorithm will choose appropriate image segmentation for various leaf sizes. Therefore we can achieve high accuracy in herbal leaf detection [22, 23].

\section{METHODOLOGIES}

Our proposed method has two-stage authentications to improve classification accuracy. Also contains phase one and two. The edge-based herbal plant detection in the first phase and classification-based herbal plant detection will be in the second phase. This proposed TSA method provides better recognition accuracy.

\subsection{Proposed Work - Phase 1}

We are using various edge detection such as Prewitt, Canny, Laplace, and Sobel edge detection operator in this proposed model. Every edge detection operator has various significant as appropriate object detection through the dimension of the object for faster identification. Generally, the image registration is responsible of purify and preprocessing input raw images.

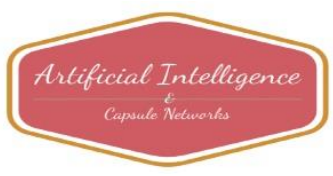


Journal of Artificial Intelligence and Capsule Networks (2021)

Vol.03/ No.02

Pages: 125-139

http://irojournals.com/aicn/

DOI: https://doi.org/10.36548/jaicn.2021.2.005

Figure 3 shows the first of the proposed model. The knowledge-based controller has chosen the edge detection operator based on measurement, which can improve the accuracy of detection process.

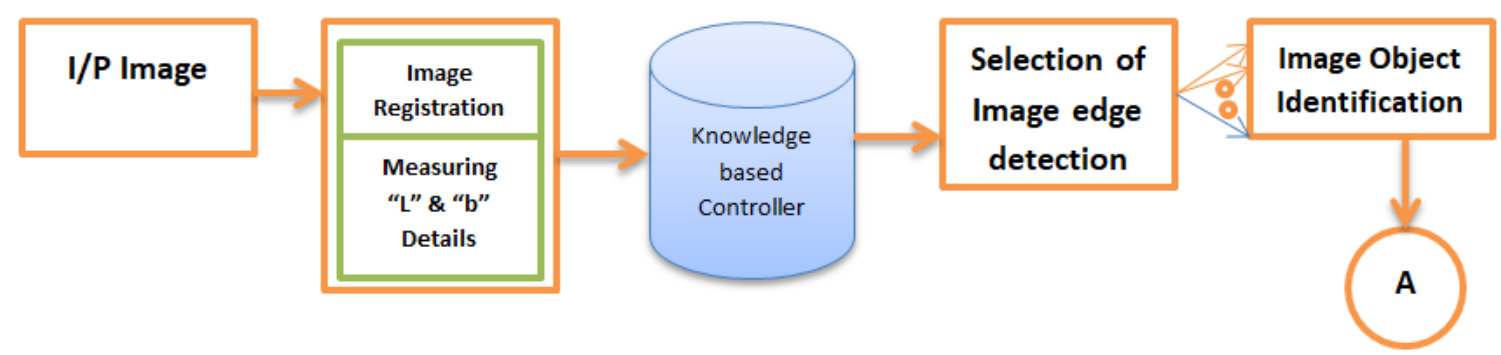

Figure 3 Proposed Work of Phase 1

\subsection{Proposed Work - Phase 2}

The input raw image will be preprocessed to improve image clarity. The colour, shape, length, and width of the pictures may all be used to extract features.

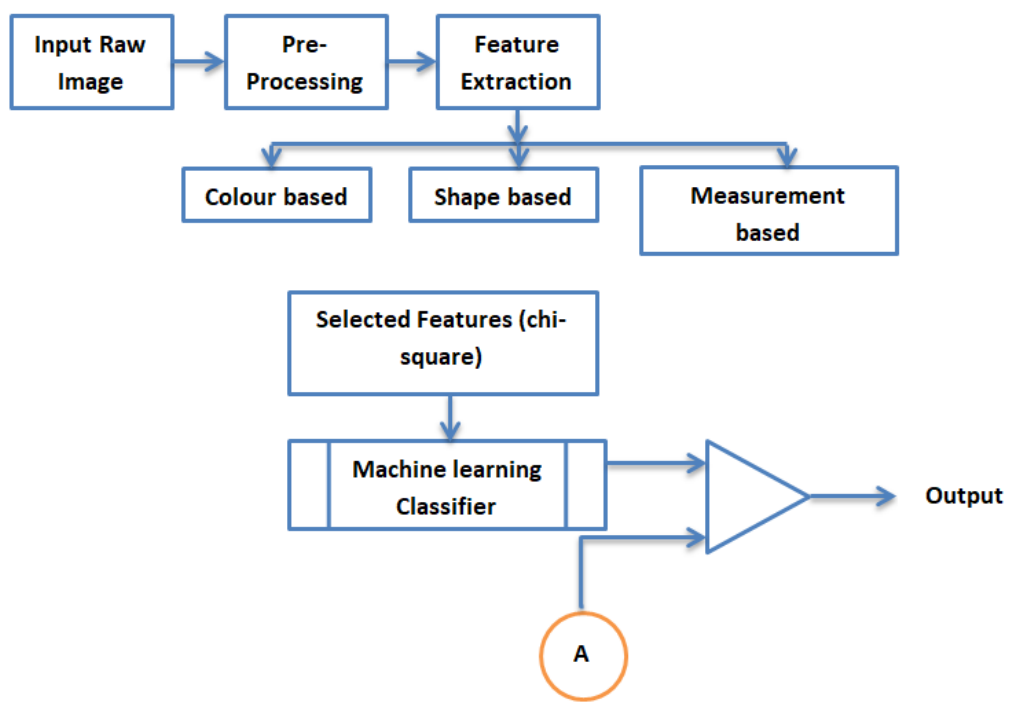

Figure 4 Proposed work of Phase 2

ISSN: 2582-2012 (online) 
Journal of Artificial Intelligence and Capsule Networks (2021)

Vol.03/ No.02

Pages: 125-139

http://irojournals.com/aicn/

DOI: https://doi.org/10.36548/jaicn.2021.2.005

This selected feature is extracted through the chi-square technique to improve the classification.

The CNN classifiers are used for the classification of input extracted images by leaf, a sample which is a recent and innovative development for the identification process. Due to the human visual system's similarity, CNN classifier may be used. Table 1 Ex-OR gate truth table

\begin{tabular}{|l|l|l|}
\hline $\mathbf{0}$ & $\mathbf{0}$ & $\mathbf{1}$ \\
\hline $\mathbf{0}$ & $\mathbf{1}$ & $\mathbf{0}$ \\
\hline $\mathbf{1}$ & $\mathbf{0}$ & $\mathbf{0}$ \\
\hline $\mathbf{1}$ & $\mathbf{1}$ & $\mathbf{1}$ \\
\hline
\end{tabular}

\section{Step 1}

Shape information through the visual cortex (V1 \& V2) by the contour of the image.

\section{Step 2}

Shape, color, and measurement of leaves sent to the tertiary visual cortex (V3).

\section{$\underline{\text { Step } 3}$}

CNN used multiple convolution filters to process features to obtain the appropriate feature vectors for perfect classification. The factorization has taken for the various parameters to improve the effectiveness of the model.

\section{$\underline{\text { Step } 4}$}

Inception structure in the pooling layers to identify the herbal plant leaf perfect.

\section{Step 5}

Compare phase 1 and phase 2 with EX-OR gate operation.

\section{$\underline{\text { Step } 6}$}

The iteration will check through 3 more loops.

\section{$\underline{\text { Step } 7}$}

The identical result is obtained by both stage identification all three times, which follows the EXOR gate operation; this complete identification is announced. If the truth table is broken, the AND gate is used. 
Journal of Artificial Intelligence and Capsule Networks (2021)

Vol.03/ No.02

Pages: 125-139

http://irojournals.com/aicn/

DOI: https://doi.org/10.36548/jaicn.2021.2.005

\section{RESULTS \& DISCUSSION}

In this study, we used 250 leaf samples with the front and back of the image. We split the dataset with $80 \% \& 20 \%$ for training and testing purposes respectively. Also, we concentrated to calculate the area, perimeter of the input images to achieve higher accuracy [24]. Figure 5 shows the accurate detection of the herbal plant lengthwise by the Sobel operator in our proposed system.
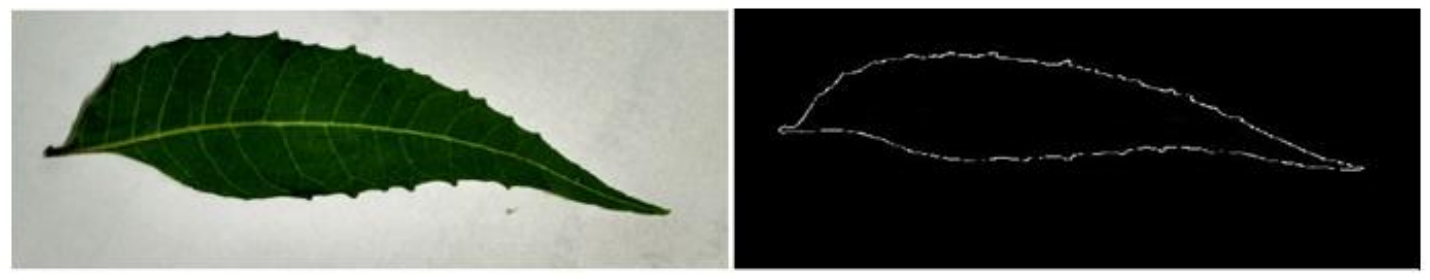

Figure 5 length wise accurate detection

The various groups of the features of herbal plant leaf structures are compared with given input plant leaves. These features are having own property of every leaf structure of a group. Our suggested approach does not account for the fact that all leaves have the same aspect ratio, centroid, and roundness. This method was successful in recognizing the leaf of a herbal plant. Recognize each distinctive characteristic of the herbal plant accurately. Figure 6 depicts the evidence of the edge detector operators' performance in a wider range of plant leaves between Canny and Sobel. Depending on these findings, we believe that edge detectors are operated based on the length and width of the leaf size, as illustrated in figure 6.

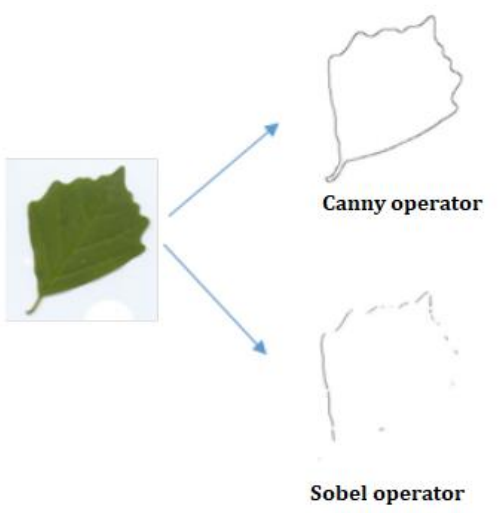

Figure 6 Obtained Results of Proposed Methodology

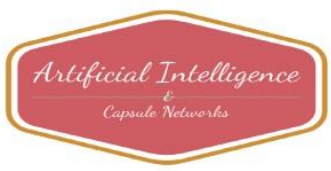


Journal of Artificial Intelligence and Capsule Networks (2021)

Vol.03/ No.02

Pages: 125-139

http://irojournals.com/aicn/

DOI: https://doi.org/10.36548/jaicn.2021.2.005

Figure 7 shows some of the obtained accurate results to compare with the existing methods. The length of object-wise accurate detection is very suitable with the Sobel operator. The herbal plant leaf identification done through canny edge detection operator is more accurate by utilizing breadth-wise detection than the Sobel operator. This variation provided good classification results in final accurate plant leaf identification.

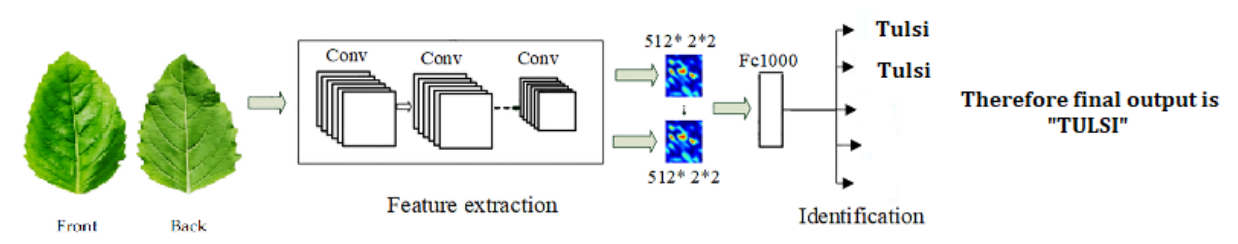

Figure 7 Overall Performance Analysis of Proposed Method

Figure 8 shows the overall performance analysis of the proposed methodology. The improvement classification result has shown in the figures and tables as well. The medicinal plant leaf datasets are divided according to size using the convergence region.

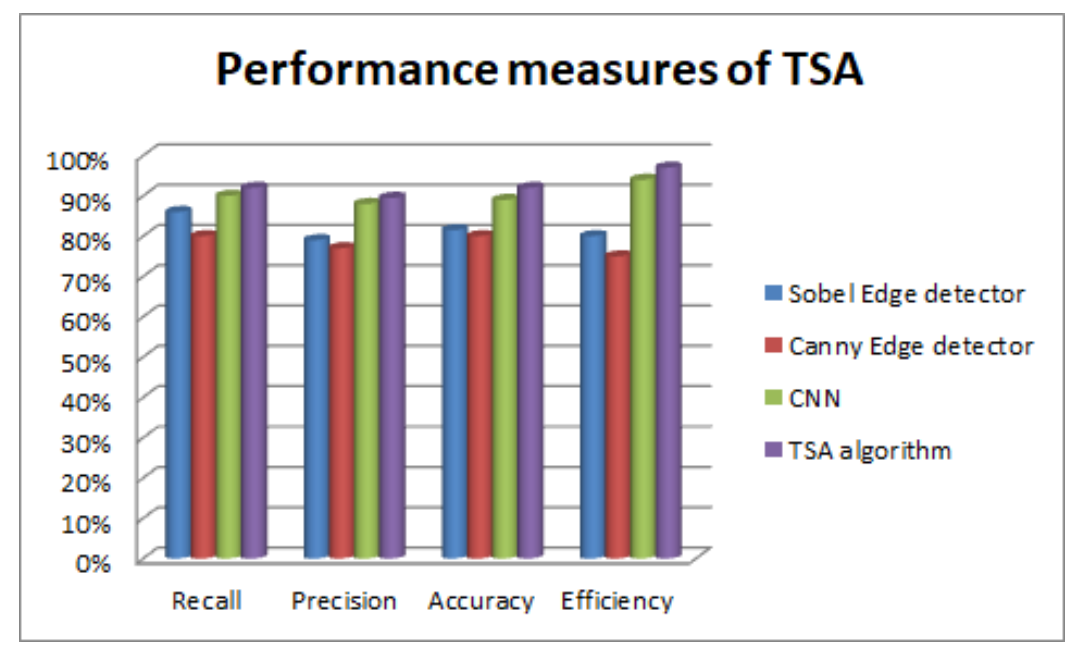

Figure 8 Overall Performance Analysis of Proposed Method

of the proposed model reaches $92 \%$ accuracy, 92\% recall, 91\% precision, and $95 \%$ efficiency of the model that shows good results comparing with existing methods. The calculated numerical performance metrics of the proposed model are tabulated in table 2. 
Journal of Artificial Intelligence and Capsule Networks (2021)

Vol.03/ No.02

Pages: 125-139

http://irojournals.com/aicn/

DOI: https://doi.org/10.36548/jaicn.2021.2.005

Table 2 performance analysis of proposed method

\begin{tabular}{|c|c|c|c|c|c|c|}
\hline S.No & Methods & Recall & Precision & Accuracy & Efficiency & $\begin{array}{c}\text { Recognition } \\
\text { error (\%) }\end{array}$ \\
\hline 1 & $\begin{array}{c}\text { Image } \\
\text { Segmentation } \\
\text { process (Sobel } \\
\text { Edge operator) }\end{array}$ & $86 \%$ & $79 \%$ & $81.5 \%$ & $80 \%$ & 0.071 \\
\hline 2 & $\begin{array}{c}\text { Canny Edge } \\
\text { detector }\end{array}$ & $80 \%$ & $77 \%$ & $80 \%$ & $75 \%$ & 0.175 \\
\hline 3 & $\begin{array}{c}\text { Single classifier } \\
\text { (CNN) }\end{array}$ & $90 \%$ & $88 \%$ & $89 \%$ & $94 \%$ & 0.053 \\
\hline 4 & $\begin{array}{c}\text { Proposed TSA } \\
\text { algorithm }\end{array}$ & $92 \%$ & $89.5 \%$ & $92 \%$ & $97 \%$ & 0.008 \\
\hline
\end{tabular}

Few leaves are equal in length and breadth while their measurements are computed. Here, the proposed model has randomly chosen the appropriate herbal plant. The classification methods are minimizing the error by using the CNN method. If the tail of the proposed system is having two or three types, which violates Ex-Or gate operation AND logic gate operation to improve the classification accuracy.

\section{CONCLUSION}

Thus, the proposed methodology has delivered good results to effectively detect the herbal plant leaf. It has proved that, the detection rate has improved than any other single tone classifiers and pure image processing techniques through two-stage verification processes. Our datasets are texture and multispectral-based one, which will support feature selection to optimize the herbal plants. This dataset is very standardized and refined through the medicinal laboratory. Our approach includes the chi-square feature selection through our standard dataset. Besides, our proposed methodology is named as two-stage authentication processes to detect the herbal plant leaves. The classification section is enhanced to improve the detection rate through our proposed methodology. This can help farmers and common people to increase the production of Ayurveda provisions. This perfect automatic classification system helps to identify the medicinal plants without getting any human support in various enterprise sectors such as botanists, taxonomists, Ayurveda manufacturing companies, and Ayurveda practitioners. Our proposed algorithm will

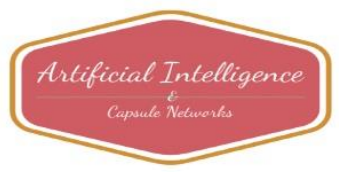


Journal of Artificial Intelligence and Capsule Networks (2021)

Vol.03/ No.02

Pages: 125-139

http://irojournals.com/aicn/

DOI: https://doi.org/10.36548/jaicn.2021.2.005

be extended to identify and classify more medicinal plants such as flowers, fruit, and seeds in an accurate manner further. This edge-based detection of medicinal plants is providing better accuracy than other existing methods. Though our proposed system provides better effectiveness, the computation time is very large and also the pixel-based process consumes more storage space in the array of the algorithm used for pixel. . To rectify those limitations of the process, we will further concentrate to work on advanced digital image processing and statistical approach of support vector machine approach as future work $[25,26]$.

\section{REFERENCES}

[1] Dhaya, R. "Flawless Identification of Fusarium Oxysporum in Tomato Plant Leaves by Machine Learning Algorithm." Journal of Innovative Image Processing (JIIP) 2, no. 04 (2020): 194-201.

[2] Dinesh Shitole, Faisal Tamboli, Krishna Motghare, Raj Kumar Raj,"Ayurvedic Herb Detection using Image Processing', International Journal of Trend in Scientific Research and Development (IJTSRD) e- ISSN: 2456 - 6470Volume: 3 | Issue: 4 | May-Jun 2019.

[3] Adam, Edriss Eisa Babikir. "Evaluation of Fingerprint Liveness Detection by Machine Learning Approach-A Systematic View." Journal of ISMAC 3, no. 01 (2021): 16-30.

[4] R Janani and A Gopal, "Identification of selected medicinal herbs leaves Using Image Features and ANN", 2013 International Conference on Advanced Electronic System (ICAES), pp 978-1-4799-1441-8.

[5] Manoharan, Samuel. "Early diagnosis of Lung Cancer with Probability of Malignancy Calculation and Automatic Segmentation of Lung CT scan Images." Journal of Innovative Image Processing (JIIP) 2, no. 04 (2020): 175-186.

[6] Wäldchen, J.; Rzanny, M.; Seeland, M.; Mäder, P. Automated plant species identificationtrends and future directions. PLoS Comput. Biol. 2018, 14, e1005993. 
Journal of Artificial Intelligence and Capsule Networks (2021)

Vol.03/ No.02

Pages: 125-139

http://irojournals.com/aicn/

DOI: https://doi.org/10.36548/jaicn.2021.2.005

[7] Hamdan, Yasir Babiker. "Faultless Decision Making for False Information in Online: A Systematic Approach." Journal of Soft Computing Paradigm (JSCP) 2, no. 04 (2020): 226-235.

[8] Turkoglu, M.; Hanbay, D. Recognition of plant leaves: An approach with hybrid features produced by dividing leaf images into two and four parts. Appl. Math. Comput. 2019, 352, 1-14.

[9] Ranganathan, G. "A Study to Find Facts Behind Preprocessing on Deep Learning Algorithms." Journal of Innovative Image Processing (JIIP) 3, no. 01 (2021): 66-74.

[10] Manojkumar P., Surya C. M., and Varun P. Gopi, "Identification of Ayurvedic Medicinal Plants by Image Processing of Leaf Samples", 2017 Third International Conference on Research in Computational Intelligence and Communication Networks (ICRCICN), pp 978-1- 5386-19315 .

[11] Dutta, Sayantan, and Ayan Banerjee. "Highly Precise Modified Blue Whale Method Framed by Blending Bat and Local Search Algorithm for the Optimality of Image Fusion Algorithm." Journal of Soft Computing Paradigm (JSCP) 2, no. 04 (2020): 195-208.

[12] Adams Begue, Venitha Kowlessur, Fawzi Mahomoodally, Upasana Singh and Sameerchand, "Automatic Recognition of Medicinal Plants using Machine Learning Techniques", International Journal of Advanced Computer Science and Applications, Vol. 8, No. 4, 2017.

[13] Sungheetha, Akey, and Rajesh Sharma. "3D Image Processing using Machine Learning based Input Processing for Man-Machine Interaction." Journal of Innovative Image Processing (JIIP) 3, no. 01 (2021): 1-6.

[14] H. X. Kan, L. Jin, and F. L. Zhou," Classification of Medicinal Plant Leaf Image Based on Multi-Feature Extraction", Pattern Recognition and Image Analysis, Vol. 27, No. 3, 2017, pp. 581-587, 1054-6618. @ Pleiades Publishing, Ltd.

[15] Palani, U., Mrs D. Vasanthi, and Ms S. Rabiya Begam. "Enhancement of Medical Image Fusion Using Image Processing." Journal of Innovative Image Processing (JIIP) 2, no. 04 (2020): 165-174.

[16] Riddhi H. Shaparia, Dr. Narendra M. Patel and Prof. Zankhana H. Shah," Flower Classification using Texture and Color Features", International Conference on Research and Innovations in Science, Engineering \&Technology, Volume 2, 2017, Pages 113-118. 
Journal of Artificial Intelligence and Capsule Networks (2021)

Vol.03/ No.02

Pages: 125-139

http://irojournals.com/aicn/

DOI: https://doi.org/10.36548/jaicn.2021.2.005

[17] Marco Seeland, Michael Rzanny, Nedal Alaqraa, Jana Wa "ldchen, Patrick Ma "der, "Plant species classification using flower images-A comparative study of local feature representations", PLOS ONE | DOI:10.1371/journal.pone.0170629 February 24, 2017.

[18] Pradeep kumar Choudhary, Rahul Khandekar, Aakash Borkar, and Punit Chotaliya, "Image processing algorithm for fruit identification", International Research Journal of Engineering and Technology (IRJET), Vol 4 Issue 3, e-ISSN: 2395 -0056, p-ISSN: 2395-0072, Mar -2017.

[19] D Venkataraman and Mangayarkarasi N, "Computer Vision Based Feature Extraction of Leaves for Identification of Medicinal Values of Plants", IEEE International Conference on Computational Intelligence and Computing Research, 978-1-5090-0612-0/16/\$31.00 @2016 IEEE.

[20] Ruaa Adeeb Abdulmunem Al-falluji, "Color ,Shape and Texture based Fruit Recognition System", International Journal of Advanced Research in Computer Engineering \& Technology (IJARCET) Volume 5, Issue 7, ISSN: 2278 - 1323 ,July 2016.

[21] Sana O M1, R.Jaya2,"Ayurvedic Herb Detection Using Image Processing”, International Journal of Computer Science and Information Technology Research, Vol. 3, Issue 4, pp: (134139), Month: October - December 2015.

[22] Dhaya, R. "Analysis of Adaptive Image Retrieval by Transition Kalman Filter Approach based on Intensity Parameter." Journal of Innovative Image Processing (JIIP) 3, no. 01 (2021): 7 20.

[23] Hang, J.; Zhang, D.; Chen, P.; Zhang, J.;Wang, B. Classification of Plant Leaf Diseases Based on Improved Convolutional Neural Network. Sensors 2019, 19, 4161

[24] Chen, Joy Iong Zong, and Lu-Tsou Yeh. "Analysis of the Impact of Mechanical Deformation on Strawberries Harvested from the Farm." Journal: Journal of ISMAC September 2020, no. 3 (2020): 166-172

[25] Lukic, M.; Tuba, E.; Tuba, M. Leaf recognition algorithm using support vector machine with Hu moments and local binary patterns. In Proceedings of the 2017 IEEE 15th International 
Journal of Artificial Intelligence and Capsule Networks (2021)

Vol.03/ No.02

Pages: 125-139

http://irojournals.com/aicn/

DOI: https://doi.org/10.36548/jaicn.2021.2.005

Symposium on Applied Machine Intelligence and Informatics, Herl0any, Slovakia, 26-28

January 2017; pp. 000485-000490.

[26] Yasir Babiker Hamdan et al "Construction of Statistical SVM based Recognition Model for Handwritten Character Recognition" published in Journal of Information Technology and Digital World (2021) Vol. 03/ No. 02 Pages: 92-107.

\section{Author's Biography}

J. Samuel Manoharan, is a professor in the Department of Electronics and Communication Engineering, in Sir Isaac Newton College of Engineering and Technology, India. His area of research are Digital Image and Signal Processing, Data Security and Cryptography, Embedded Systems, Biomedical Instrumentation, Artificial Intelligence, Robotics, Deep Learning, Cognitive Science, Ad-hoc Networks, Artificial Neural Network, Evolutionary Computing, Speech Recognition and Autonomous Systems.

ISSN: 2582-2012 (online) 